\title{
The Analysis of an HIV and AIDS Empowering Group Work Programme for Adolescents: A Quantitative Evaluation
}

\author{
K. Olivier ${ }^{1}$, H. Strydom ${ }^{2}$ \\ ${ }^{1}$ Social Worker in Private Practice, Leeudoringstad, South Africa; ${ }^{2}$ Potchefstroom Campus of the North-West University, Potchef- \\ stroom, South Africa. \\ Email: herman.strydom@nwu.ac.za
}

Received January $11^{\text {th }}, 2013$; revised February $21^{\text {st }}, 2013$; accepted February $28^{\text {th }}, 2013$

\begin{abstract}
In this article an HIV and AIDS group work programme empowering adolescents to deal with the possible death of their parents/caregivers will be evaluated in a quantitative fashion. The data were obtained by means of the Child Functioning Inventory High School (CFI-HIGH) developed by Perspective Training College and the Generalized Contentment Scale (GCS) of Hudson (Bloom et al., 1999: 220). The guidelines for selection and inclusion of respondents in the comparison and experimental groups will be discussed, followed by information on the measuring instruments. The article will conclude with conclusions and recommendations.
\end{abstract}

Keywords: Evaluation; HIV and AIDS; Empowering Group Work Programme; Adolescents

\section{Introduction}

The aim of this article is to evaluate and determine the quantitative effect of a compiled group work programme on adolescents from households infected with and affected by HIV and AIDS. This programme is based on the following theoretical assumption: adolescents can be empowered to deal with the possible death of their parents/caregivers by means of an HIV and AIDS group work programme. The empowerment of adolescents through this programme includes aspects such as a healthy lifestyle, identity and self-esteem, roles and relationships, effective communication, assertiveness, and conflict management. Other aspects included are problem solving, decision making and time management, coping with stress and emotions, orientation to and implications of AIDS, spirituality and death as a reality, bereavement, financial security and planning for the future.

\section{Problem Statement}

Responses to adolescents affected by HIV and AIDS should address their needs [1] and programme development must be done in response to adolescents' needs via their active participation in the entire process [2]. The exploration of these needs should form the foundation of an effectively designed group work programme to act in the best interest of the adolescent [2,3]. For adolescents to reach their optimal potential as adults, it is important that they should be guided through a programme empowering them with skills within their specific circumstances [4].

To determine whether the designed empowering group work programme as a social intervention has produced the intended results, it is necessary to evaluate it [5]. Programme evaluation includes the systematic collection of information on programme activities, characteristics and outcomes of the programme to make judgements concerning the programme, improvement of programme effectiveness and making suggestions regarding the future use of the programme. In the evaluation of a programme as a potential method, it should be borne in mind that it should produce the information required to assess the programme's progress towards achieving its desired outcomes [6].

\section{Objective}

The objective of this article was to evaluate the effectiveness of the developed empowering group work programme by way of standardised measuring instruments in order to focus on the quantitative findings of this study.

\section{Research Methodology}

The intervention research model (D \& D) was utilised for this study. D \& D is a phase model consisting of six 
phases [7]. The fifth phase of this model, namely evaluation and advanced development, was utilised. For the experiment the comparison group pretest-posttest design was utilized [8]. This design includes two groups, namely the experimental group and the comparison group. According to this design only the experimental group received the programme, with the same before and after test for both groups, which were the Child Functioning Inventory High School (CFI-HIGH) [9] and the Generalized Contentment Scale (GCS) [10].

Although the assignment to the groups was done randomly as with the classic experimental design [11], the groups can never be exactly the same and therefore will be regarded as an experimental and comparative group [12]. The mentioned standardized measuring instruments were utilized in the process of the evaluation of the group work programme [13]. The aim of this experiment was to determine whether the application of an empowering group work programme had an influence on the adolescent's functioning as part of a household infected with or affected by HIV and AIDS.

A total of 24 adolescents were selected by means of accidental sampling [14]. Only 16 of these adolescents were available after the initial sampling to conduct this research. All of these 16 adolescents met the criteria for inclusion in the experiment, being adolescents (12 to 18 years) showing willingness to participate in the total programme and who are from households infected with or affected by HIV and AIDS where all of them had a parent or caretaker who is infected with HIV or is already ill due to AIDS or those adolescents who have already lost a parent(s) to AIDS. These 16 adolescents were then divided into 2 groups with age and gender as main criteria to have two as identical as possible groups. One group was then selected randomly as the comparison group and the other as the experimental group.

As ethical consideration, the comparison group was informed that they would be afforded the opportunity to undergo the same group work programme as the experimental group once the last-mentioned group had completed it. This is to give all participants equal access to an empowering opportunity, namely that participation in a research project should be a learning experience for all concerned [15].

The quantitative data in this study was statistically computed with SAS [16]. The results were interpreted, inferences made and conclusions drawn. The practical significance of results is not only important when results of the population data are reported but also for commenting on the practical significance of a statistically significant result [17].

\section{Evaluation by Means of Child Functioning Inventory High School (CFI-HIGH)}

The Child Functioning Inventory High School (CFI-
HIGH) was administered in pre- and post-testing as part of the quantitative evaluation. The CFI-HIGH is a standardized pen-and-paper self-reporting measuring instrument that can be utilized to evaluate the general functioning of children of high school age (adolescents) regarding various aspects. The following areas of personal functioning are included: Positive functioning areas (including perseverance, satisfaction, future perspective), Self-perception (including anxiety, guilt feelings, lack of self-worth, isolation, responsibility for others, lack of assertiveness), Trauma dynamics (including memory loss, frustration, helplessness, attitude towards adults, mistrust, stigma, body image, personal boundaries, school problems), Relationships (including relationship with friends, relationship with mother, relationship with father, relationship with family), Decision making skills (including independency, responsibility).

\subsection{Reliability of the CFI-HIGH}

The Cronbach Alpha coefficient is a criterion to determine the internal consistency of a measuring instrument [18]. The reliability of the CFI-HIGH of Perspective was calculated with Cronbach Alfa coefficient. Huysamen [18] points out that a reliability coefficient as low as 0.6 can be considered acceptable for making decisions regarding groups. Four of the constructs were found to be not reliable with reliability coefficients that were below 0.5 . The constructs of the CFI-HIGH measuring anxiety, responsibility, relationships with friends and independency, can thus not be accepted as reliable. The constructs of the CFI-HIGH measuring perseverance, satisfaction, future perspective, guilt feelings, lack of self-worth, isolation, lack of assertiveness, memory loss, frustration, helplessness, attitude towards adults, mistrust, stigma, body image, personal boundaries, school problems, relationship with mother, relationship with father, relationship with family and responsibility were found to be reliable with reliability coefficients that varied between 0.57 and 0.90 .

\subsection{Pre- and Post-Testing}

Both the comparison and experimental groups were requested to complete the CFI-HIGH one week before the onset of the group work programme. After the pre-testing, only the experimental group was exposed to the group work programme consisting of 12 sessions presented over a period of six weeks. The post-test was conducted by requesting both the experimental and the comparison group to complete the CFI-HIGH once again. This post-testing took place one week after the experimental group had completed the group work programme. Because of the small sample size, the tests might not have had enough power to indicate statistical significance on a $5 \%$ level of significance, and effect sizes will be used as an indication of the practical significance of differences. 
The results are expounded in Table 1.

\subsection{Pre- and Post-Test Results}

\subsubsection{Results after Pre-Testing}

After pre-testing, the p-value $(\mathrm{P})$ of the grouping of the experimental and comparison group regarding all the constructs measured $>0.05$. P-values $>0.05$ indicate that the two groups did not differ statistically significantly on a $5 \%$ significance level at this point with regard to these constructs. The effect-size (D) of the test between groups (after pre-testing) regarding future perspective, guilt feelings, lack of self-worth, lack of assertiveness, memory loss, helplessness, mistrust, stigma, relationship with father, relationship with family and responsibility were found to be $>0.4$, which indicates a medium visible and significant difference and an effect size $>0.8$ indicates that there is a large visibly to practically significant difference between the two groups regarding these constructs before the experimental group was exposed to the programme, with the experimental group being better. The effect-size (D) of the test between groups (after pretesting) regarding isolation, frustration, attitude towards adults, body image, personal boundaries, school problems and relationship with mother was found to be $<0.4$, which indicates an insignificant difference between the two groups regarding these constructs before the experimental group was exposed to the programme.

\subsubsection{Results after Post-Testing}

- Within experimental group

After post-testing, the p-value (P) of the experimental group regarding the constructs of satisfaction, guilt feelings

Table 1. Results of pre- and post-testing of CFI-HIGH.

\begin{tabular}{|c|c|c|c|c|c|c|c|c|c|c|c|c|c|c|}
\hline & \multicolumn{6}{|c|}{ Experimental group } & \multicolumn{6}{|c|}{ Comparison group } & \multirow{2}{*}{\multicolumn{2}{|c|}{$\begin{array}{c}\text { Ancova } \\
\text { Test between } \\
\text { groups }\end{array}$}} \\
\hline & \multicolumn{2}{|c|}{ Pre-Test } & \multicolumn{2}{|c|}{ Post-Test } & \multicolumn{2}{|c|}{$\begin{array}{l}\text { Test with } \\
\text { in group }\end{array}$} & \multicolumn{2}{|c|}{ Pre-Test } & \multicolumn{2}{|c|}{ Post-Test } & \multicolumn{2}{|c|}{$\begin{array}{c}\text { Test } \\
\text { with in group }\end{array}$} & & \\
\hline & Average & $\begin{array}{l}\text { Standard } \\
\text { deviation }\end{array}$ & Aveage & $\begin{array}{l}\text { Standard } \\
\text { deviation }\end{array}$ & $\mathrm{P}$ & $\mathrm{D}$ & Average & $\begin{array}{l}\text { Standard } \\
\text { deviation }\end{array}$ & Average & $\begin{array}{r}\text { Standard } \\
\text { deviation }\end{array}$ & $\mathrm{P}$ & $\mathrm{D}$ & P-value & Effect-size \\
\hline Perseverance & 85.1 & 20.0 & 92.5 & 13.2 & 0.1705 & 0.37 & 60.1 & 24.3 & 63.5 & 22.1 & 0.2849 & 0.14 & 0.0616 & -1.19 \\
\hline $\begin{array}{c}\text { Future } \\
\text { perspective }\end{array}$ & 70.6 & 19.9 & 74.3 & 11.0 & 0.5557 & 0.19 & 50.1 & 18.5 & 46.9 & 15.5 & 0.3566 & -0.17 & 0.0094 & 1.74 \\
\hline Guilt feelings & 28.8 & 25.7 & 18.0 & 17.9 & 0.0176 & -0.42 & 42.0 & 22.3 & 42.8 & 17.3 & 0.7416 & 0.04 & $<0.0001$ & 3.28 \\
\hline $\begin{array}{c}\text { Lack of } \\
\text { self-worth }\end{array}$ & 19.1 & 15.9 & 9.9 & 9.6 & 0.0460 & -0.58 & 36.3 & 22.1 & 34.5 & 24.4 & 0.5062 & -0.08 & 0.0642 & 1.12 \\
\hline Isolation & 43.6 & 20.5 & 40.1 & 15.5 & 0.3001 & -0.17 & 41.3 & 20.1 & 41.4 & 22.2 & 0.9581 & 0.00 & 0.3983 & 0.45 \\
\hline $\begin{array}{c}\text { Lack of } \\
\text { assertiveness }\end{array}$ & 29.4 & 22.9 & 19.8 & 20.6 & 0.0705 & -0.42 & 40.4 & 23.3 & 41.1 & 22.6 & 0.7043 & 0.03 & 0.0271 & 1.27 \\
\hline Memory loss & 23.9 & 28.5 & 13.9 & 18.5 & 0.1460 & -0.35 & 35.9 & 22.8 & 39.9 & 24.9 & 0.2524 & 0.18 & 0.0160 & 1.39 \\
\hline Frustration & 28.1 & 26.7 & 14.9 & 11.1 & 0.2003 & -0.49 & 38.8 & 29.8 & 39.3 & 33.1 & 0.8679 & 0.02 & 0.0697 & 0.97 \\
\hline Helplessness & 33.9 & 26.5 & 21.0 & 13.8 & 0.1038 & -0.49 & 46.4 & 19.9 & 46.8 & 23.9 & 0.8904 & 0.02 & 0.0190 & 1.34 \\
\hline $\begin{array}{c}\text { Attitude } \\
\text { towards adults }\end{array}$ & 23.5 & 26.6 & 15.3 & 12.6 & 0.2619 & -0.31 & 33.6 & 21.8 & 35.4 & 19.2 & 0.4496 & 0.08 & 0.0105 & 1.48 \\
\hline Mistrust & 45.4 & 12.7 & 32.5 & 7.1 & 0.0362 & -1.02 & 53.4 & 7.4 & 51.0 & 9.5 & 0.1572 & -0.32 & 0.0027 & 2.00 \\
\hline Stigma & 44.5 & 26.6 & 31.6 & 18.5 & 0.0685 & -0.48 & 57.8 & 17.1 & 60.5 & 15.7 & 0.2846 & 0.16 & 0.0021 & 1.95 \\
\hline Body image & 26.3 & 29.2 & 14.4 & 18.9 & 0.0540 & -0.41 & 32.4 & 25.6 & 33.1 & 25.9 & 0.1970 & 0.03 & 0.0058 & 1.66 \\
\hline $\begin{array}{c}\text { Personal } \\
\text { boundaries }\end{array}$ & 32.3 & 25.1 & 12.5 & 6.2 & 0.0495 & -0.79 & 34.4 & 16.0 & 41.8 & 18.2 & 0.1464 & 0.46 & 0.0005 & 2.33 \\
\hline $\begin{array}{l}\text { Relationship } \\
\text { with mother }\end{array}$ & 74.6 & 26.4 & 80.5 & 16.6 & 0.4645 & 0.22 & 66.0 & 29.0 & 59.3 & 26.5 & 0.0412 & -0.23 & 0.0379 & 1.17 \\
\hline $\begin{array}{l}\text { Relationship } \\
\text { with father }\end{array}$ & 58.5 & 25.4 & 63.1 & 28.2 & 0.2713 & 0.18 & 40.9 & 23.6 & 41.0 & 23.0 & 0.9751 & 0.00 & 0.3930 & 0.48 \\
\hline $\begin{array}{l}\text { Relationship } \\
\text { with family }\end{array}$ & 77.4 & 17.0 & 85.0 & 18.5 & 0.1017 & 0.45 & 50.8 & 27.6 & 48.8 & 27.2 & 0.3699 & -0.07 & 0.0604 & 1.18 \\
\hline Responsibility & 71.3 & 19.6 & 83.5 & 18.7 & 0.0249 & 0.62 & 59.8 & 24.2 & 59.8 & 23.1 & 1.0000 & 0.00 & 0.0131 & 1.50 \\
\hline
\end{tabular}


mistrust and responsibility measured $<0.05$, which indicates a statistically significant difference. The p-value $(\mathrm{P})$ after post-testing of the experimental group regarding perseverance, future perspective, lack of self-worth, isolation, lack of assertiveness, memory loss, frustration, helplessness, attitude towards adults, stigma, body image, personal boundaries, school problems, relationship with mother, relationship with father and relationship with family measured $>0.05$, which indicates that there is no statistically significant difference on a $5 \%$ significance level regarding these constructs in this group after their exposure to the programme with regard to these constructs. The effect-size (D) of the experimental group regarding guilt feelings, lack of self-worth, lack of assertiveness, frustration, helplessness, stigma, body image, school problems, relationship with family and responsibility was found to be $>0.5$, which indicates a medium visible improvement. The effect-size (D) of the experimental group regarding satisfaction, mistrust and personal boundaries measured $>0.7$, which indicates that there is a large practically significant improvement in the experimental group after being exposed to the programme. The effect-size (D) of experimental group regarding perseverance, future perspective, isolation, memory loss, frustration, attitude towards adults, relationship with mother and relationship with father is $<0.4$, which indicates that there is no visibly significant difference in the experimental group after their exposure to the programme regarding these constructs.

- Within comparison group

After post-testing, the only p-value $(\mathrm{P})<0.05$ of the comparison group is regarding relationship with mother with the $p$-value $=0.04$. It indicates a statistically significant difference regarding this construct. All the other constructs measured $>0.05$. P-values that are $>0.05$ indicate that there is no statistically significant difference on a 5\% significance level in the comparison group after post-testing. The effect-size (D) of the comparison group regarding all constructs measured $<0.5$, which indicates that there is no practical or significant improvement in the comparison group after post-testing.

- Test between groups

After post-testing, the p-value (P) of the test between groups (experimental and comparison groups) regarding satisfaction, future perspective, guilt feelings, lack of assertiveness, memory loss, helplessness, attitude towards adults, mistrust, stigma, body image, personal boundaries, school problems, relationship with mother and responsibility measured $<0.05$, which indicates a statistically significant difference regarding the ANCOVA means (adjusted for pre-test counts) of these constructs between the two groups. All the other constructs, including perseverance, lack of self-worth, isolation, frustration, relationship with father and relationship with family, measured $p>0.05$. P-values that are $>0.05$ indicate that there is no statistically significant difference between these groups on a 5\% significance level. The effect-size (D) of the ANCOVA test between groups (experimental and control groups) regarding perseverance, satisfaction, future perspective, guilt feelings, lack of self-worth, lack of assertiveness, memory loss, frustration, helplessness, attitude towards adults, stigma, body image, personal boundaries, school problems, relationship with mother, relationship with family and responsibility are all $>0.8$. An effect size $>0.8$ indicates that there is a large practically significant difference between the groups. The effect-size (D) of ANCOVA test between groups regarding isolation and relationship with father is 0.45 and 0.48 . An effect-size of 0.5 indicates that there is a medium visibly significant difference on the test between the groups regarding these constructs. In all these differences the experimental group was better than the comparison group in the post-test.

\subsubsection{Results of Constructs}

The following aspects were observed regarding the results within the constructs of the CFI-HIGH:

\section{- Perseverance}

In both the experimental group and the comparison group there was no statistically significant improvement with regard to pre- and post-testing. In the test between groups there was no statistically significant difference between the groups with regard to perseverance. In the post-testing there was a large practically significant improvement regarding perseverance.

\section{- Satisfaction}

With regard to satisfaction, the experimental group showed a statistically significant improvement in the group and the comparison group showed no statistically significant improvement in the group. In the test between groups there was a large practically significant difference regarding satisfaction, where adjusted means of the $e x-$ perimental group were better than those of the comparison group.

- Future perspective

After pre-testing (before the experimental group was exposed to the programme), the grouping of the experimental and comparison groups showed no statistically significant difference between the groups. In the grouping of the experimental and comparison groups there was a large practically significant difference between the groups. In both the experimental group and the comparison group there was no statistically significant improvement with regard to pre- and post-testing. There was a large practically significant improvement in the test between groups regarding future perspective, where adjusted means of the experimental group were better than those of the comparison group. 
- Guilt feelings

After pre-testing, the grouping of the experimental and comparison groups showed no statistically significant difference between the groups. In the grouping of the experimental and comparison groups there was a medium visibly significant improvement between the groups after pre-testing. The experimental group showed a statistically significant improvement with regard to guilt feelings and the comparison group showed no statistically significant improvement in the group. In the test between groups there was a large practically significant improvement regarding guilt feelings, where adjusted means of the experimental group were better than those of the comparison group.

- Lack of self-worth

After pre-testing, the grouping of the experimental and comparison groups showed no statistically significant difference between the groups regarding lack of selfworth. In the grouping of the experimental and comparison groups there was a large practically significant difference between the groups after pre-testing. The $e x-$ perimental group showed a statistically significant improvement in the group and the comparison group showed no statistically significant improvement in the group. In the test between groups there was a large practically significant improvement regarding lack of selfworth, where adjusted means of the experimental group were better than those of the comparison group.

- Isolation

After pre-testing, the grouping of the experimental and comparison groups showed no statistically significant difference between the groups. In the grouping of the experimental and comparison groups there was no visibly significant difference between the groups after pretesting. Neither the experimental group nor the comparison group showed any statistically significant improvement in the groups with regard to pre- and post-testing. In the test between groups there was no practically significant improvement regarding isolation.

- Lack of assertiveness

After pre-testing, the grouping of the experimental and comparison groups showed no statistically significant difference between the groups. In the grouping of the experimental and comparison groups there was no visible and significant difference between the groups after pre-testing. Neither the experimental group nor the comparison group showed any statistically significant improvement in the groups with regard to pre- and posttesting. In the test between groups there is a large practically significant improvement regarding lack of assertiveness, where adjusted means of the experimental group were better than those of the comparison group.

- Memory loss

After pre-testing, the grouping of the experimental and comparison groups showed no statistically significant difference between the groups. In the grouping of the experimental and comparison groups there was no visible and significant difference between the groups after pre-testing. Neither the experimental group nor the comparison group showed any statistically significant improvement in the groups with regard to pre- and posttesting. In the test between groups there was a large practically significant improvement regarding memory loss, where adjusted means of the experimental group were better than those of the comparison group.

- Frustration

After pre-testing, the grouping of the experimental and comparison groups showed no statistically significant difference between the groups. The grouping of the experimental and comparison groups showed no visible and significant difference between the groups after pretesting. Neither the experimental group nor the comparison group showed any statistically significant improvement in the groups with regard to pre- and post-testing. In the test between groups there was a large practically significant improvement regarding frustration, where adjusted means of the experimental group were better than those of the comparison group.

- Helplessness

After pre-testing, the grouping of the experimental and comparison groups showed no statistically significant differences between the groups. In the grouping of the experimental and comparison groups there was no visibly significant difference between the groups after pretesting. Neither the experimental group nor the comparison group showed any statistically significant improvement in the groups with regard to pre- and post-testing. There was a large practically significant improvement regarding helplessness in the test between groups, where adjusted means of the experimental group were better than those of the comparison group.

- Attitude towards adults

After pre-testing, the grouping of the experimental and comparison groups showed no statistically significant difference between the groups. In the grouping of the experimental and comparison groups there was no visible and significant difference between the groups after pre-testing. Neither the experimental group nor the comparison group showed any statistically significant improvement in the groups with regard to pre- and posttesting. There was a large practically significant improvement regarding attitude towards adults in the test between groups, where adjusted means of the experimental group were better than those of the comparison group.

- Mistrust

After pre-testing, the grouping of the experimental and comparison groups showed no statistically significant 
difference between the groups. In the grouping of the experimental and comparison groups there was a medium visibly significant difference between the groups after pre-testing. The experimental group showed a statistically significant improvement with regard to pre- and post-testing and the comparison group showed no statistically significant improvement in the groups with regard to pre- and post-testing. In the test between groups there was a large practically significant improvement regarding mistrust, where adjusted means of the experimental group were better than those of the comparison group.

- Stigma

After pre-testing, the grouping of the experimental and comparison groups showed no statistically significant difference between the groups. In the grouping of the experimental and comparison groups there was a medium visibly significant difference between the groups after pre-testing. Neither the experimental group nor the comparison group showed any statistically significant improvement in the groups with regard to pre- and posttesting. In the test between groups there was a large practically significant improvement regarding stigma, where adjusted means of the experimental group were better than those of the comparison group.

- Body image

After pre-testing, the grouping of the experimental and comparison groups showed no statistically significant difference between the groups. In the grouping of the experimental and comparison groups there was no visibly significant difference between the groups after pretesting. Neither the experimental group nor the comparison group showed any statistically significant improvement with regard to pre- and post-testing. In the test between groups there was a large practically significant difference regarding body image, where adjusted means of the experimental group were better than those of the comparison group.

- Personal boundaries

After pre-testing, the grouping of the experimental and comparison groups showed no statistically significant differrence between the groups. In the grouping of the experimental and comparison groups there was no visibly significant difference between the groups after pretesting. The experimental group showed a statistically significant improvement and the comparison group showed no statistically significant improvement with regard to personal boundaries. In the test between groups there was a large practically significant improvement regarding personal boundaries, where adjusted means of the experimental group were better than those of the comparison group.

- School problems

After pre-testing, the grouping of the experimental and comparison groups showed no statistically significant difference between the groups. In the grouping of the experimental and comparison groups there was no visible and significant difference between the groups after pre-testing. The experimental group showed a statistically significant improvement in the group with regard to school problems. The comparison group showed no statistically significant improvement in the group with regard to school problems. In the test between groups there was a large practically significant improvement regarding school problems, where adjusted means of the experimental group were better than those of the comparison group.

- Relationship with mother

After pre-testing, the grouping of the experimental and comparison groups showed no statistically significant difference between the groups. In the grouping of the experimental and comparison groups there was no visibly significant difference between the groups after pretesting. The experimental and comparison groups both showed statistically significant improvement with regard to pre- and post-testing. In the test between groups there was a large practically significant improvement regarding relationship with mother, where adjusted means of the experimental group were better than those of the comparison group.

- Relationship with father

After pre-testing, the grouping of the experimental and comparison groups showed no statistically significant difference between the groups. In the grouping of the experimental and comparison groups there was a medium visibly significant difference between the groups after pre-testing. Neither the experimental group nor the comparison group showed any statistically significant improvement with regard to pre- and post-testing. In the test between groups there was no visibly significant difference regarding relationship with father.

- Relationship with family

After pre-testing, the grouping of the experimental and comparison groups showed no statistically significant difference between the groups. In the grouping of the experimental and comparison groups there was a medium visibly significant difference between the groups after pre-testing. Neither the experimental group nor the comparison group showed any statistically significant improvement with regard to pre- and post-testing. In the test between groups there was a large practically significant improvement regarding relationship with family, where adjusted means of the experimental group were better than those of the comparison group.

- Responsibility

After pre-testing, the grouping of the experimental and comparison groups showed no statistically significant difference between the groups. In the grouping of the experimental and comparison groups there was no visi- 
bly significant difference between the groups after pretesting. The experimental group showed a statistically significant improvement in the group with regard to responsibility. The comparison group showed no statistically significant improvement in the group with regard to responsibility. In the test between groups there was a large practically significant improvement regarding responsibility, where adjusted means of the experimental group were better than those of the comparison group.

\section{Evaluation by Means of Generalized Contentment Scale (GCS)}

The Generalized Contentment Scale (GCS) of Hudson [10] was administered during pre- and post-testing. The GCS is a standardized pen-and-paper self-reporting measuring instrument designed to measure the way respondents feel about their life and surroundings. In this experiment, the aim of administering the GCS was to determine the respondents' general contentment with life. Both the pre- and post-test data were utilized to determine a link between the respondents' contentment with life and the impact of an empowering group work programme. The GCS scores range from $0-100$ and the following values were used to interpret the GCS scores:

- A score higher than 35 shows need for improvement.

- A score between 25 and 35 indicates warning area that needs attention.

- A score below 25 is in the recommended range.

\subsection{Reliability of the GCS}

The Cronbach Alpha coefficient is a criterion to determine the internal consistency of a measuring instrument [18]. The reliability of the GCS of Hudson was calculated with Cronbach Alpha coefficient and was 0.79. Huysamen [18] points out that a reliability coefficient as low as 0.6 is acceptable to make decisions regarding groups. All the tests (constructs) of the GCS can be accepted as reliable.

\subsection{Pre- and Post-Testing}

Both the comparison group and the experimental group were requested to complete the GCS one week before the onset of the group work programme. After the pre-testing, only the experimental group were exposed to the group work programme which was presented over a period of six weeks including 12 sessions. The post-test was conducted by requesting both the experimental group and the comparison group to complete the GCS once again. This post-testing took place one week after the experimental group had completed the group work programme. Because of the small sample size, the tests might not have had enough power to indicate statistical significance on a $5 \%$ level of significance, and effect sizes were used as an indication of the practical significance of differences. The results are expounded in Table 2.

\subsection{Pre- and Post-Test Results}

\subsubsection{Results after Pre-Testing}

After pre-testing, the p-value $(\mathrm{P})$ of the grouping of the experimental and comparison groups measured 0.20. This p-value is $>0.05$ and indicates that the two groups did not significantly differ statistically on a $5 \%$ significance level at this point. After pre-testing, the effect-size (D) of the grouping of the experimental and comparison groups was 0.84 . The experimental group was better than the comparison group.

\subsubsection{Results after Post-Testing}

- Within experimental group

After the experimental group had been exposed to the programme, a p-value of 0.004 was measured. A p-value of $<0.05$ indicated that there is a statistically significant difference in this group after their exposure to the programme. The effect-size (D) of the experimental group after their exposure to the programme was 1.45. An effect-size $>0.8$ indicates that there is a visibly and practically significant improvement in the group after their exposure to the programme. The experimental group improved regarding their generalized contentment after their exposure to the programme.

- Within comparison group

After the post-testing of the comparison group, a p-value of 0.0322 was measured. A p-value of $<0.05$ indicates that there is a statistically significant difference in this group after the exposure of the experimental group to the programme. The effect-size (D) of the comparison group after post-testing is 0.27 . An effect-size $<0.5$ indicates that there was a no visibly significant improvement in this group after the post-testing.

Table 2. Results of pre- and post-testing of GCS.

\begin{tabular}{|c|c|c|c|c|c|c|c|c|c|c|c|c|c|c|}
\hline & \multicolumn{6}{|c|}{ Experimental group } & \multicolumn{6}{|c|}{ Comparison group } & \multirow{2}{*}{\multicolumn{2}{|c|}{$\begin{array}{c}\text { Ancova } \\
\text { Test between groups }\end{array}$}} \\
\hline & \multicolumn{2}{|c|}{ Pre-Test } & \multicolumn{2}{|c|}{ Post-Test } & \multicolumn{2}{|c|}{$\begin{array}{c}\text { Test } \\
\text { within group }\end{array}$} & \multicolumn{2}{|c|}{ Pre-Test } & \multicolumn{2}{|c|}{ Post-Test } & \multicolumn{2}{|c|}{$\begin{array}{l}\text { Test within } \\
\text { group }\end{array}$} & & \\
\hline $\begin{array}{c}\text { General } \\
\text { contentment }\end{array}$ & 39.1 & 15.1 & 17.3 & 8.4 & 0.004 & 1.45 & 48.9 & 13.8 & 52.9 & 11.8 & 0.0322 & 0.29 & $<0.0001$ & 4.0 \\
\hline
\end{tabular}




\section{- Test between groups}

After the experimental group had been exposed to the programme, the ANCOVA test between the groups (experimental and comparison groups) registered a p-value of $<0.0001$. A $p$-value of $<0.05$ indicates that there is a statistically significant difference regarding the $\mathrm{AN}$ COVA means (adjusted for pre-test counts) of this construct between the two groups. The effect-size (D) of the ANCOVA test between groups (experimental and comparison groups) after the experimental group had been exposed to the programme, is 4.00 . An effect-size $>0.8$ indicates that there is a visible and significant improvement between the groups after post-testing. The experimental group improved regarding their generalized contentment, but the comparison group did not show any visibly significant improvement.

\section{Discussion}

The reliability of the CFI-HIGH was calculated and 20 of the 24 constructs were regarded as reliable. After the pre-testing the grouping of the experimental and comparison groups showed no statistical differences. There were visibly significant differences in more than half of the constructs in the test between the groups before the experimental group was exposed to the programme. After the programme intervention, the experimental group showed practically significant difference regarding guilt feelings, lack of self-worth, lack of assertiveness, frustration, helplessness, stigma, body image, school problems, relationship with family, responsibility, satisfaction, mistrust and personal boundaries.

Within the comparison group, in all the constructs but one, relationship with mother, there was no statistically significant difference. There are no visibly significant differences in any of the constructs in the comparison group after post-testing. In 14 of the 20 reliable constructs, a statistically significant difference in the test between groups was measured. In all of the constructs, excluding isolation and relationship with father, in the test between groups, there was a practically significant difference after the experimental group had been exposed to the programme.

The experimental group improved in all but two of the constructs, but with no visibly significant difference the comparison group did not improve in any of the constructs. All the tests of the GCS were accepted as reliable. After pre-testing the two groups showed no statistically significant differences. There was a large practically significant difference between the two groups after pre-testing, with the experimental group being the better of the two. After the experimental group had been exposed to the programme, there was a statistically significant difference in the group. There was a large practically significant difference in this group after the programme intervention.

In the comparison group there was a statistically significant difference in the group after post-testing. There was no visibly significant difference in the comparison group after post-testing. There was a statistically significant difference between the experimental and comparison groups after the programme intervention in the test between groups. A large practically significant difference was reported in the test between groups after programme intervention. The experimental group improved regarding their generalized contentment, but the comparison group showed no visibly significant difference, which indicates no improvement.

\section{Recommendations}

Based on the discussion of the results from this study and the conclusion drawn, the following recommendations can be made:

- This empowerment programme should be evaluated with larger and more groups, like the Solomon-four group design, so that the quantitative results of this research can be confirmed or refuted.

- Respondents find it difficult to answer the CFI-HIGH in English (for most respondents their second or third language) and it was completed as a group with a translator translating every statement from English to Tswana. The CFI-HIGH can be more user-friendly and accessible if the questionnaire and answering sheets are translated in more languages such as Tswana, isiXhosa and isiZulu.

- The understanding, perception and knowledge base of an adolescent of 12 years and one of 18 years differ considerably, based on their levels of development and life experience. Much time was spent explaining concepts to the younger members of the group and the older adolescents felt obliged to take care and help them with various activities during the programme. It is recommended for future presentation of the programme that the age distribution of the adolescents should be closer, say 15 - 19 years.

\section{Conclusion}

The purpose of this study was to evaluate and determine the effect of a compiled group work programme on adolescents from households infected with and affected by HIV and AIDS. Adolescents for inclusion in the experimental and comparison groups were selected by means of accidental sampling from a limited population. After sampling, the adolescents were divided into two groups randomly according to gender and age. For this experiment the comparison group pretest-posttest design was utilized and included two groups, namely an experimental group and a comparison group. For the aim of evalua- 
tion the two groups were quantitatively evaluated by means of the CFI-HIGH of Perspective Training College and the GCS of Hudson.

\section{REFERENCES}

[1] M. Ungar, "Handbook for Working with Children and Youth: Pathways to Resilience across Cultures and Context," 2005.

[2] N. M. Kandasamy, "Guidelines for a Social Work Programme for Adolescents in the North-West Province," M.A. Dissertation, Potchefstroom University for Christian Higher Education, Potchefstroom, 2002.

[3] H. E. Scholtz, "Guidelines for Social Group Work with Adolescents from Problem Families," M.A. Dissertation, Potchefstroom University for Christian Higher Education, Potchefstroom, 1998.

[4] L. Van Heerden, "The Development and Evaluation of a Life Skills Programme to Enhance the Social Functioning of the Early Black Adolescent," M.A. Dissertation, North-West University, Potchefstroom, 2001.

[5] C. B. Fouche and A. S. De Vos, "Problem Formulation," In: A. S. De Vos, Ed., Research at Grass Roots: For the Social Sciences and Human Service Professions, 3rd Edition, Van Schaik, Pretoria, 2005, pp. 100-110.

[6] P. Rankin, M. L. Weyers and M. Williams, "The Use of the Programme Logic Model (PLM) in Structured Social Work Interventions: Theory and Practice," The Social Work Researcher-Practitioner, Vol. 20, No. 3, 2008, pp. 334-357.

[7] A. S. De Vos, "Intervention Research," In: A. S. De Vos, Ed., Research at Grass Roots: For the Social Sciences and Human Service Professions, 3rd Edition, Van Schaik, Pretoria, 2005, pp. 392-407.
[8] R. M. Grinnell, "Social Work Research and Evaluation," F.E. Peacock Publishers, New York, 2001.

[9] Perspective Training College, "Perspective and Walmyr Assessment Scales," Perspective Training College, Potchefstroom, 2006.

[10] M. Bloom, J. Fischer and J. G. Orme, "Evaluating Practice: Guidelines for the Accountable Professional," Allyn \& Bacon, Needham Heights, 1999.

[11] R. M. Grinnell and M. Williams, "Research in Social Work: A Primer," F.E. Peacock Publishers, New York, 1990.

[12] A. Rubin and E. R. Babbie, "Research Methods for Social Work," Thomson Brooks/Cole, London, 2005.

[13] C. S. L. Delport, "Quantitative Data-Collection Methods," In: A. S. De Vos, Ed., Research at Grass Roots: For the Social Sciences and Human Service Professions, 3rd Edition, Van Schaik, Pretoria, 2005, pp. 159-191.

[14] H. Strydom, "Sampling and Sampling Methods," In: A. S. De Vos, Ed., Research at Grass Roots: For the Social Sciences and Human Service Professions, 3rd Edition, Van Schaik, Pretoria, 2005, pp. 192-204.

[15] H. Strydom, "Ethical Aspects of Research in the Social Sciences and Human Service Professions," In: A. S. De Vos, Ed., Research at Grass Roots: For the Social Sciences and Human Service Professions, 3rd Edition, Van Schaik, Pretoria, 2005, pp. 56-70.

[16] SAS Institute Inc., "The SAS System for Windows Release 9.1 TS Level 1 M3," SAS Institute Inc., Cary, 2003.

[17] S. M. Ellis and H. S. Steyn, "Practical Significance (Effect Sizes) Versus or in Combination with Statistical Significance (P-Values)," Management Dynamics, Vol. 12, No. 4, 2003, pp. 51-53.

[18] G. K. Huysamen, “Sielkundige Meting:'n Inleiding," 2nd Edition, Van Schaik, Pretoria, 1996. 